\title{
Configuration of Own PBX System within a Campus Area Network and Implementation of VoWi-Fi
}

\author{
Md. Nahid Akter Shafi and Abdullah Al Kawser
}

\begin{abstract}
VoIP is a technology that is popular all over the world for its cost effectiveness. Asterisk is an open source, converged telephony platform that supports all the protocols of voip (sip, iax, h.323, mgcpetc). We establish this voip based asterisk pbx on ubuntu 11.10 (a linux-based os) which allow us to make unlimited free calls with the help of ip phone or traditional phone within a lan/can without internet connection. Wi-fi is often presented as a model of successful unlicensed frequency spectrum deployment. For wireless communication, we extend our voip based pbx to wi-fi technology, with the name vowi-fi. The convergence time depend on the speech content e.g. voiced/unvoiced. In terms of unvoiced segments, the convergence time is stable as in voiced phases it fluctuates. It is also rely on silence suppression. But it associate degree bound at the top of the segment. As most of the internet subscribers of Asian countries use very low internet bandwidth. Hence, the goal of this analysis is to propose to use it without internet. In a real life environment it is tested practically in research that it is possible to transmit voice satisfactorily described in this paper.
\end{abstract}

Index Terms-VoIP, asterisk PBX, Wi-Fi, IP phone, VoWi-Fi.

\section{INTRODUCTION}

Once The great think about Voice over Internet Protocol (VoIP) technology for which people are so massively turning to is to make calls cheaper or completely free. Day by day, the VoIP technology is developing as user friendly. Our objective is to focus this technology and to introduce Mobile VoIP to make people realize about the beauty, strength, and opportunity of this technology.

The drawbacks of traditional telephone system are inflexible, complicated and expensive. These are designed to the vendor's need, not us. Many telephone operators still build inflexible systems, extremely expensive, running complicated, ancient code on impressively engineered yet obsolete hardware. Basically, this incredible flexibility comes with a huge price. The powerful asterisk is just a candle in the darkness.

Asterisk is an open source platform, far more than just a Private Branch Exchange (PBX) in software that provides all the functionality of a traditional telephone system with greater flexibility [1]-[3]. The Internet telephony (IP telephony) has been presented as a technology that can replace existing fixed-line services and disrupt the telecommunications industry by offering new low-priced

Manuscript received September 2, 2015; revised November 11, 2015.

Md. Nahid Akter Shafi is with the Network Operations Center of Inspire Systems Limited in Dhaka, Bangladesh (e-mail: nahid_apee@yahoo.com).

Abdullah Al Kawer is with the Information technology department of Dhaka Power Distribution Company Limited (DPDC) in Dhaka, Bangladesh (e-mail: kawser.telecom@gmail.com). services [4].

Asterisk PBX can be used for IP telephony. We establish asterisk PBX on a Linux-based operating system Ubuntu and the system is so designed so that it is possible to make unlimited free calls within a Local Area Network (LAN) without internet connection.

The 802.11b Wi-Fi technology uses unlicensed or license free radio frequency spectrum [5]. We extend our PBX to Wi-Fi technology for wireless connectivity so that callers can make VoIP calls using Wi-Fi supported mobile phones, laptop or any other devices that have Wi-Fi support within the hotspot with no internet connection. It is as so named VoWi-Fi.

\section{MethodOLOGY}

Asterisk can be configured in any modern computer that runs on a Linux system. We configure the PBX asterisk 1.8 on Ubuntu 11.10 machine. The basic connection of our PBX system is shown in Fig. 1.

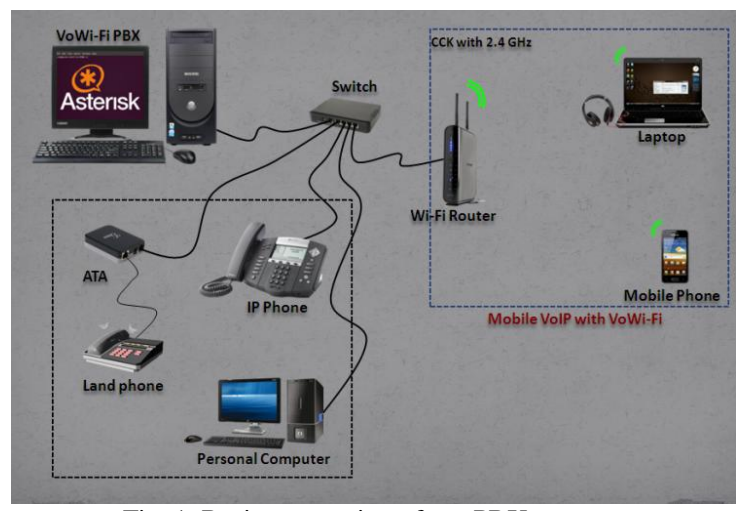

Fig. 1. Basic connection of our PBX system.

Let $\mathrm{x}(\mathrm{t})$ denote the voice signal with unwanted noise at bit rate 5.3 is fed to the $\mathrm{H}$ maps, the output will generate a signal (let's denote $y(t)$ ) will have improved voice quality with the samIt is possible to make free calls with video support within a Local Area Network (LAN). To make calls from one party to another party, the possible options are (i) IP Phone (ii) Analog Telephone Adapter (ATA) with traditional phone (iii) Computer with softphone (a softphone is a software program that provides telephone functionality rather than using dedicated hardware) (iv) Wi-Fi supported mobile phone (Android).

Asterisk-based telephony system integrates almost all the protocols of VoIP (IAX, SIP, H.323, MGCP etc) and supports various codecs required to run a PBX system. Codecs (Compression/Decompression) are basically various mathematical tools used to encode or compress analog signals into digital bit streams with Pulse Code Modulation 
(PCM) technique using 8 bit encoding and $8 \mathrm{KHz}$ sampling, resulting $64 \mathrm{kbps}$ of data with excellent voice quality. The original PCM at $64 \mathrm{kbps}$ is G.711 which is supported by all VoIP devices. G.711 (A-Law, $\mu$-Law) codec provides better voice quality with less bandwidth usages and ensures lossless data compression. Codec H.263 is used for video call support in asterisk PBX [6]. The whole process is illustrated in Fig. 2.

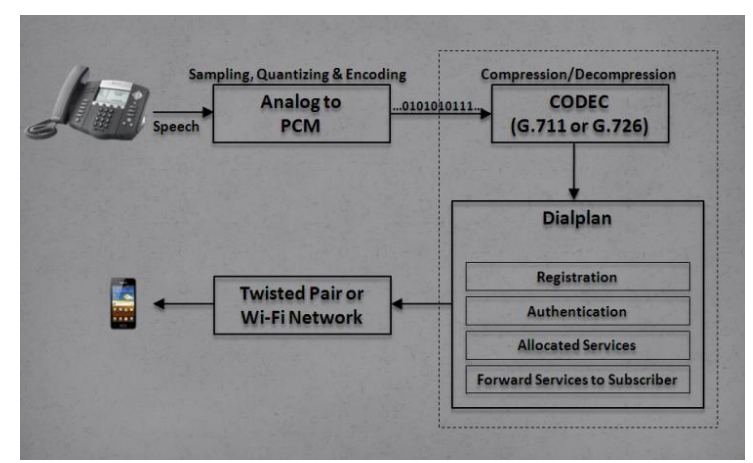

Fig. 2. Call process in asterisk PBX.

The Wi-Fi router takes the VoIP packets, decodes them, then re-encodes them into a wireless radio wave signal and broadcasts it into the air. Typically the transmitting frequency is $2.4 \mathrm{GHz}$ and it uses the modulation technique called Complementary Code Keying (CCK). Wi-Fi enabled laptop or mobile picks up the signal and receives the call.

When a call is established, a channel brings the call to the asterisk PBX [7], converting the analog signal to a digital format using codecs and translating it over a packed-based TCP/IP network. If packets are lost or delayed, there will be an interruption between the communications. Each call is received or placed on a distinct channel. Channels are used to connect VoIP protocols they support in asterisk PBX. Transmission Control Protocol (TCP) is used for call control and setup but User Datagram Protocol (UDP) is used for the voice transmission itself.

A typical Wi-Fi router can cover a range of 150 feet $(46 \mathrm{~m})$ for indoors and 300 feet $(92 \mathrm{~m})$ for outdoors which actually varies due to the physical location of the router and depends on various conditions of the environment such as obstructions and radio interference. However, two or more routers can be configured to provide hand-off support like cellular communication and also to enhance the coverage area of the network.

Each device must be given a unique IP address either from Dynamic Host Configuration Protocol (DHCP) or statically to identify the device within the network. Private IP addresses which are reserved by Internet Assigned Numbers Authority (IANA) are used in the network in order to avoid confliction with the public IP addresses used in the internet. The number of users in the PBX depends on total number of maximum hosts in different private IP address classes (Class A, Class B \& Class C).

The dialplan is basically the heart of asterisk PBX which actually defines how asterisk will handle the incoming and outgoing calls. The dialplan logic allows us to call between the extensions. The dialplan logical code is specified in different configuration files in asterisk system to authenticate the callers and to provide different services such as call holding, call waiting, voice mail, call conference etc. Unlike traditional telephone system, asterisk can provide any extension of numbers for users also extensions can have names as well. Asterisk can also solve the problem to make calls to the public traditional telephone/cellular network from the extensions by having a license and an account from telephony provider with internet connection.

Voice over Wireless Networks has the same issues as VoIP. Nature of Voice, and Scalability are imperative to the utilization of VoIP. Notwithstanding these issues, Quality of Service, Mobility, and Security of telephony activity are more hazardous in wireless networks. These issues or problems are presently being investigated for wired and wireless networks in general. These arrangements are being received inside of executions of VoIP and VoWiFi items. As more items are made for telephony over data networks, more arrangements are included by making new protocols and administrations that keep up voice traffic [8].

Several techniques have been invented for measuring the quality of the voice signal that has been processed by different compression algorithms (CODECs). One of the standard techniques for measuring quality of voice CODECs, which is also an ITU standard, is called Mean Opinion Score (MOS). MOS values, which are subjective and expressed by humans, range from 1 (worst) to 5 (perfect or equivalent to direct conversation). Table I displays some of the ITU standard CODECs and their corresponding bandwidth requirements and MOS values.

MOS is an ITU standard method of measuring voice quality based on the judgment of several participants; therefore, it is a subjective method. Table I displays each of the MOS ratings along with its corresponding interpretation, and a description for its distortion level. It is noteworthy that an MOS of 4.0 is deemed to be Toll Quality [9].

TABLE I: MEAN OPTION SCORE

\begin{tabular}{|c|c|c|}
\hline Rating & Speech Quality & Level of Distortion \\
\hline 5 & Excellent & Imperceptible \\
\hline 4 & Good & Just perceptible but not annoying \\
\hline 3 & Fair & Perceptible but slightly annoying \\
\hline 2 & Poor & Annoying but not objectionable \\
\hline 1 & Unsatisfactory & Very annoying and objectionable \\
\hline
\end{tabular}

A standout amongst the most widely recognized approaches to decide the nature of a codec is to decide the Mean Opinion Score (MOS). The MOS is a quality somewhere around one and five. A score of one means there is a correspondences breakdown. A brilliant score is a five. This is identical to an immaculate AM radio gathering. Current area line telephony accomplishes a score of four. The closer a codec is to a score of four speaks to a little contrast between the codec and current area line frameworks.

\section{SPECTROGRAM}

The spectrogram of the speech signal is an intensity plot of short time Fourier transforms (STFT) magnitude. STFT is a sequence of FFTs of windowed data segments where the windows are usually allowed to overlap in time. The data to be broken up into frames, which usually overlap to reduce artifacts at the boundary and then each frame, is Fourier transformed using K-point FFT [10]. The STFT procedure is shown in Fig. 4.

The short time Fourier transform (STFT) is expressed as: 


$$
S\left(\omega_{k}, l\right)=\sum_{n=0}^{N-1} x[n+l] \omega[n] e^{-j \omega_{k} n}
$$

where $\omega_{k}=\frac{2 \pi k}{N T}, k=0 \ldots K-1$ at given sampling frequency $f_{s}, x(n)$ is the time domain signal and $\omega(n)$ is the window function.

\section{RESULT AND DISCUSSION}

The signal has silence suppression and the related SNR can be decreased up to a sensible quality. If the output signal with reduced bit rate is proceed thru amplification process up to $5.3 \mathrm{kbps}$, we can accomplish impalpable the voice quality.

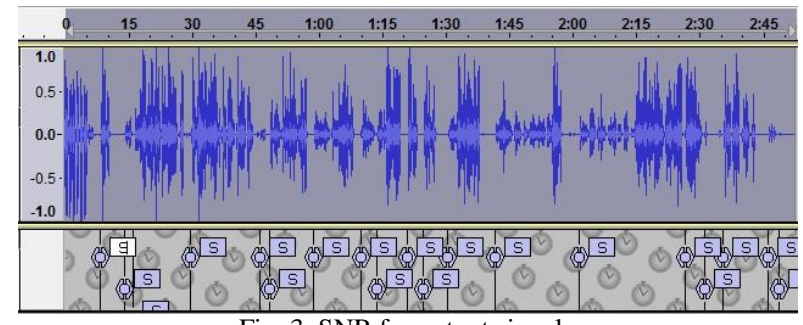

Fig. 3. SNR for output signal.

Fig. 4 shows that Codec G729 has jig gage bandwidth graph but it is good voice at desirable bandwidth.

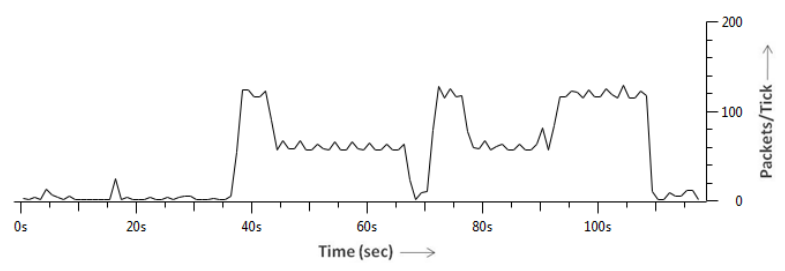

Fig. 4. Voice quality bandwidth graph for codec G729.

The spectrogram gives a compelling portrayal of the time assortment of the frequency analysis $\log$ of voice. The spectrogram of output signal is shown in Fig.5. The periods where voice is accessible can be obviously perceived from the pitch structure of the vocal line excitation. Truth be told taking a gander at histogram, and relying upon the pitch structure of speech, it is conceivable to physically section the signal into voice periods with ease and with a high level of precision.

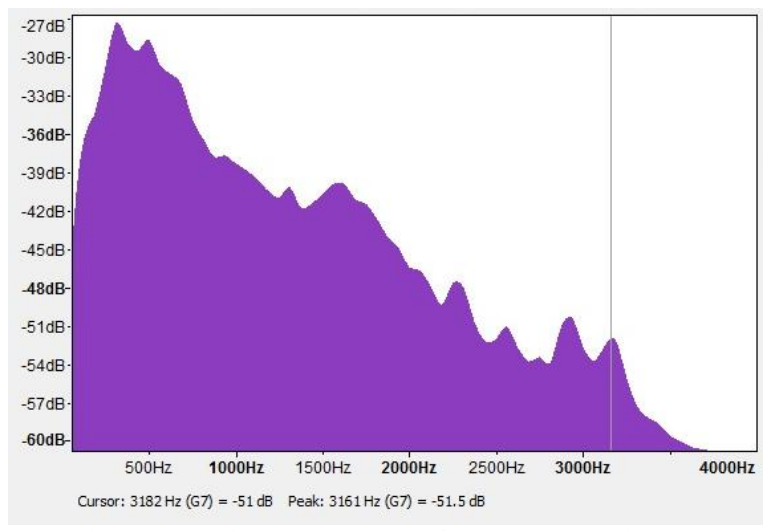

Fig. 5. Input signal spectrogram frequency analysis log.

This is no longer possible to transfer a call from our PBX to traditional Public Switched Telephone Network (PSTN) or Global Systems for Mobile communications (GSM) networks. Since we are no sharing any government resources (i.e. gateway, server or exchange), our VoIP PBX system is not illegal at all.

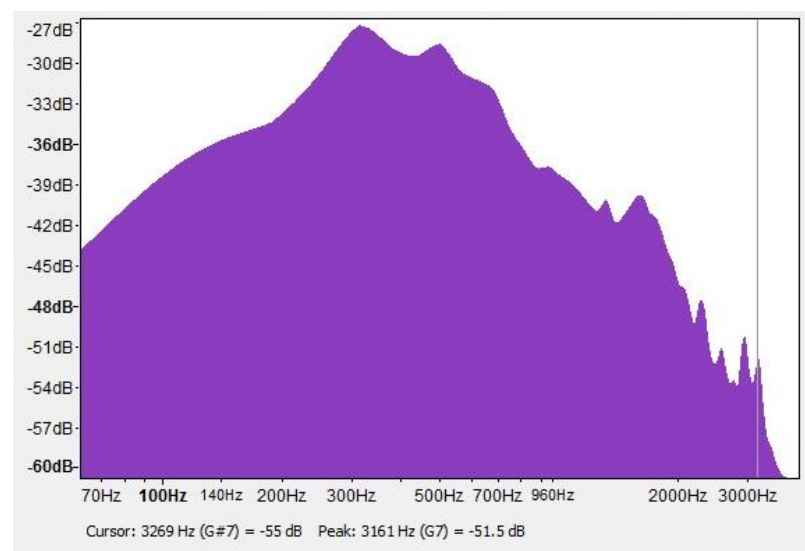

Fig. 6. Output signal spectrogram frequency analysis log.

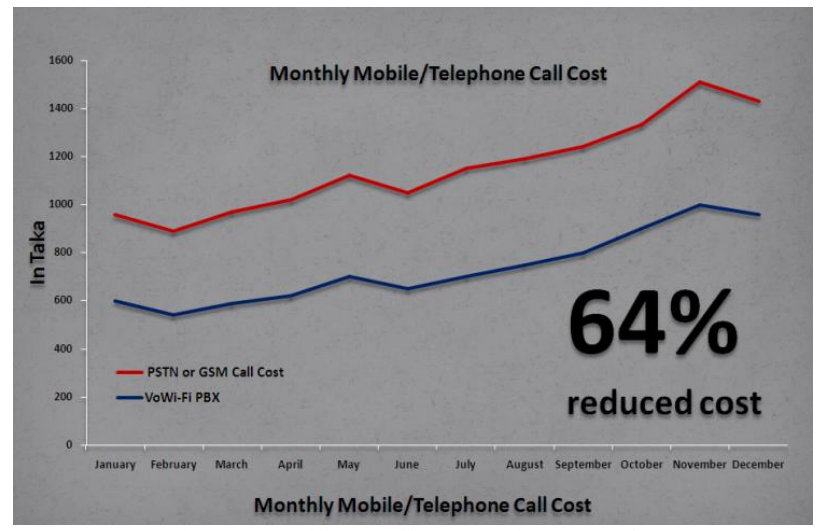

Fig. 7. Monthly mobile reduced cost graph.

This PBX allows us to make free calls, in some cases video call without internet connection within a limited geographical area such as university, hotel, school, academic building or office building where the networking equipment and transmission media are almost completely owned. Hence, it is possible to reduce the monthly telephone or mobile bill one has to pay every month.

\section{CONCLUSIONS}

Our PBX, an integration of VoIP and Wi-Fi, VoWi-Fi allows us to make unlimited free calls as well as video calls to be made over an IP network within a Campus Area Network (CAN) without internet connection. Most of the office network and campus area network system has a network foundation of switches and routers for internet usages as well as for other sharing purposes which is locally maintained and operated. Our PBX can be implemented in such a network without any interruption. Thus, one can talk freely being staying within the network rather than paying any price for it. This study is applicable particularly in LAN region. The real time implementation of voice quality had been offered through practical examination and the spectral analysis of speech signal was carried out in this work to assess the execution of voice quality investigation. The spectral analysis results are related to that of the target estimation results for the designed VoIP system. To improve the execution of the framework, these debasement parameters ought to be appropriately addressed. In future, the study 
could be used for upgrading the VoIP speech signal quality using distinctive signal processing and filtering calculations performed at higher frequency digital signal processing algorithms.

\section{FUTURE WORK}

The development of VoIP is very fast and it is implementing in mobile phones too. The telephone operators can adopt this technology which gives an enormous competitive advantage rather than confusing, expensive hardwires. Also, Asterisk can be the bridge between the traditional circuit-switched telecommunication networks with packet-switched data networks.

\section{APPENDIX}

Various dialer, SQL database, firewall system, CISCO router \& switch, OS, Ubuntu server etc.

\section{REFERENCES}

[1] S. P. Olejniczak and K. Brady, Asterisk for Dummies, John Wiley \& Sons, 2007.

[2] V. Meggelen et al., Asterisk: The Future of Telephony, O'Reilly Media, Inc., 2007.

[3] 74 Open Source Voip Apps \& Resources, December 25, 2007.

[4] I. D. Constantio, A. Papazafeiropoulou, and Y. K. Dwivedi, "The diffusion of IP telephony and vendors' commercialization strategies," Journal of Information Technology, 2009.

[5] J. F. Kurose and K. W. Ross, "Computer networking," pp. 539-540, 2007.

[6] J. V. Meggelen, L. Madsen, and J. Smith, Asterisk ${ }^{\mathrm{TM}}$ : The Future of Telephony, Second Edition, pp. 185-206, 2007.
[7] Asterisk+channels. http://www.voip-info.org/wiki/view/Asterisk+channels

[8] Vowifiside. http://www.networkworld.com/research/2004/0503vowifiside.html?p age $=1$

[9] J. V. Meggelen, L. Madsen, and J. Smith, CCNP ONT Official Exam Certification Guide, pp. 24-25, 2008.

[10] T. F. Quatieri, "Discrete-time speech signal processing: Principle and Practice, MIT Lincoln Laboratory, Liexington, Massachusetts, Prentice Hall, 2002.

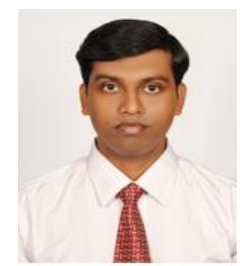

Md. NahidAkter Shafi was born in Rajshahi, Bangladesh on July 23, 1985. He received the B.Sc and M.Sc. degrees from the Department of Applied Physics and Electronic Engineering, Rajshahi University, Rajshahi, Bangladesh, in 2006 and 2007 respectively. He also certified on ITIL-Foundation, CEH, RHCE and CCNA. He has been working as a senior system engineer in the Network Operations Center of Inspire Systems Limited in Dhaka, Bangladesh since June 2011. $\mathrm{He}$ is a member of the American Society for Research (ASR), Science and Engineering Institute (SCIEI) \& International Association of Computer Science and Information Technology (IACSIT). His current research interests include VOIP, Information Security and spintronics.

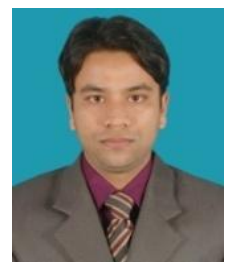

AbdullahAl Kawser was born in Dinajpur, Bangladesh on March 8, 1989. He received bachelor degree from HajeeMohammad Danesh Science \& Technology University, Dinajpur in the Department of Telecommunication \& Electronic Engineering (TEE) and master degree from Dhaka University in the Department of Information Technology. He has been working as an assistant engineer in the Dhaka.

Power Distribution Company Limited (DPDC) in Dhaka, Bangladesh since June 2015. His interests are in telecommunications and VoIP communications 